\title{
Systemic Onset Juvenile Idiopathic Arthritis (sJIA) or Still's Disease: A Diagnostic Challenge; while Presented as Fever of Unknown Origin. 2 Cases
}

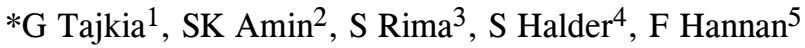

\begin{abstract}
Systemic-onset juvenile idiopathic arthritis (sJIA) or Still's disease is a chronic inflammatory disease of unknown etiology belongs to the group of Juvenile Idiopathic Arthritis. In contrast to other JIA patients in whom the joint disease usually overshadows the more general symptomatology, in Systemic-onset juvenile idiopathic arthritis (sJIA) extra-articular features such as spiking fever, hepatosplenomegaly, lymphadenopathy, rash, pleurisy, or pericarditis, and vasculopathy are most prominent. Thus the onset of disease can be vary nonspecific and may suggest bacterial or viral infection, malignancy or other rheumatic disease. As it is highly characterized by its extra-articular systemic illness features, in some ways, it resembles a fever of unknown origin. We present 2 cases of fever of unknown origin, initially presented with fever and other extra-articular features, without any arthritis, but after several months develops arthritis and finally diagnosed as Systemic-onset juvenile idiopathic arthritis (sJIA) or Still's disease .
\end{abstract}

Key Words: Fever of unknown origin, Systemic onset juvenile idiopathic arthritis, Still's disease, Juvenile Idiopathic Arthritis.

\section{Introduction}

Fever among children not only shoots up the temperature of child but also the anxiety of parents and pressure on the treating pediatrician to diagnose the condition as soon as possible. Fever beyond 2 weeks or for the practical purposes, FUO still remains the daunting challenge for any pediatrician. ${ }^{1}$ The term fever of unknown origin came into light after the publication by Petersdorf and Beeson, defined it as temperature $>38.3^{\circ} \mathrm{C}$ on several occasions and lasting longer than 3 weeks with a diagnosis which remain uncertain after 3 weeks of evaluation as an outpatient or after 1 week of evaluation in the hospital. ${ }^{2}$ Most fevers of unknown or unrecognized origin results from atypical presentations of common diseases and in some cases, the presentation as an FUO is characteristic of the disease, such as Juvenile idiopathic arthritis, but the definitive diagnosis can be established only after prolong observation because initially there are no associated or specific findings on physical examination and all laboratory results are negative or normal. ${ }^{3}$ The etiologies of FUO were classified into 5 groups namely infection, malignancy, connective tissue disorder, miscellaneous and undiagnosed. The above categorization of outcomes was based on the popular study conducted by Petersdorf and Beeson where the results came as infection (36\%), malignancy (19\%), rheumatological problems (15\%), miscellaneous $(23 \%)$ and undiagnosed $(7 \%){ }^{2}$ Most of the studies done subsequent to Petersdorf and Beeson over various time periods and at various geographical

$1 *$ Dr. Gule Tajkia, Assistant Professor, Department of Pediatrics, Anwer khan Modern Medical College \& Hospital e-mail:dr.guletajkia@gmail.com

2Prof. Syed Khairul Amin, Professor, Department of Pediatrics, Anwer khan Modern Medical College \& Hospital

${ }^{3}$ Dr. Shamim Rima, Assistant Professor, Department of Radiology \& Imaging, Anwer Khan Modern Medical College \& Hospital

${ }^{4}$ Dr. Soma Halder, Registrar, Department of Pediatrics, Anwer khan Modern Medical College \& Hospital

${ }_{5}^{5}$ r. Fabia Hannan, Medical Officer, Department of Pediatrics, Anwer khan Modern Medical College \& Hospital

*Corresponding Author

Date of submission: 08.08.2018 Date of acceptance: 13.09 .2018

AKMMC J 2019; 10(1) : 84-88 
areas revealed infections as the major cause of FUO in both children and adult population. Rheumatologic or Connective tissue disorder is the third common cause. ${ }^{4-6}$ Among the Connective tissue disorder Systemic-onset juvenile idiopathic arthritis is always a possibility in such child diagnosis, which may be difficult as all manifestations may not present at same time. Ten percent of patients may present with extra-articular manifestations only and may not develop arthritis for many months. At onset extra-articular manifestations including rash, fever, lymphadenopathy, hepatosplenomegaly and serositis predominates. However, diagnosis is often challenging, since the disease can mimic infections and malignancies. ${ }^{7,8}$

\section{Case Reports}

\section{Case 1}

A 10 months old female child presented with high grade fever for 1month duration with maculopapular fine rash on trunk and extremities whenever she got fever and disappear as fever subside. On detailed enquiry, parents gave no history of cough, respiratory difficulty, joint swelling or restriction of joint movement, redness of eyes, swelling at neck, dysuria or bleeding from any site. She was immunized as per national immunization schedule with normal developmental history. For these complaints she was investigated and treated in other hospital as indoor patient for 15 days where she received broad spectrum antibiotic along with intravenous fluid and paracetamol as antipyretic. She came to our hospital as there was no improvement of fever. On admission she had high grade fever (1030 F) with maculopapular rash, tachycardia, generalized lymphadenopathy, hepatomegaly $(4 \mathrm{~cm}$ below costal margin) with firm in consistency, spleen just palpable. The patient's laboratory tests showed severe anaemia, neutrophilic leukocytosis, thrombocytopenia with high ESR and high CRP. Urine routine examination, Widal test, blood culture, tuberculin test, bone marrow examination were done which were normal. USG Abdomen showed hepatosplenomegaly. Malaria antigen test, ANA, Anti ds DNA, RF Test, Anti-CCP antibody were negative. She was started IV Ceftriaxone but fever continued so IV Meropenam was added later. As fever continued and not responding to antibiotic and considering the typical high-spiking fever classically with 2 peaks daily (Double quotidian) associated with rash, hepatosplenomegaly sJIA was in suspicion, though the child not fulfill the diagnostic criteria of sJIA as the patient had no arthritis. Then we started Naproxen, after that fever subsided and patient was discharged and advised for follow up. After 5 months, she again admitted with fever, pain and swelling of multiple joints. This time she has full blown feature of arthritis involving small joints of hands and feet, both wrist joints, ankle joints, joints of cervical spine. As the patient already received naproxen, now we give methotraxate along with prednisolone and NSAID which brings her in remission and she can walk and play again.

\section{Case 2}

A 4 years old boy of nonconsanguineous marriage presented with a 3 weeks history of daily spiking fever, fatigue, weight loss and poor general condition. He also had severe body ache and joint pain but there was no joint swelling or effusion. He had no history of travelling or residing in malaria or kala-azar endemic zone or no history of contact with tubercular patient. On examination he was febrile at $38.6^{\circ} \mathrm{C}$, with an ill appearance and irritable. He had generalized lymphadenopathy and hepatosplenomegaly but there was no bleeding manifestation and no arthritis. Our first suspicion was Leukemia. Investigations showed anemia with lymphocytic leukocytosis without any blast cell, high ESR, platelet was normal, Widal and malarial antigen was negative, Dengue and Chikungunya antibody was negative. ANA, Anti ds DNA, AntiCCP antibody, RF Test were negative. USG Abdomen showed hepatosplenomegaly. Bone marrow examination was normal. His fever was unresponsive to antibiotic and antipyretic (Paracetamol). By this time the parents became so much anxious and feared of malignancy as the investigation reports were inconclusive, so they went to Singapore. After 2 months he came to OPD with fever and arthritis of multiple joints and finally diagnosed as Systemic-onset juvenile idiopathic 
arthritis. This time he was treated with Naproxen and Ranitidine. In next follow up his clinical condition was improved than before.

\section{Discussion}

Despite the improvement in diagnostic techniques, the fever of unknown origin (FUO) remains a challenge to overcome, comprising $50 \%$ of the cases without clear etiology 9 . Systemic-onset juvenile idiopathic arthritis (sJIA), sometimes called Still's disease, is a systemic inflammatory disease classified within the spectrum of juvenile idiopathic arthritis (JIA), one of the cause of PUO. Systemic-onset juvenile idiopathic arthritis (sJIA), as a subtype includes about $10-15 \%$ of all JIA patients, but the percentage is higher when severe JIA cases are considered ${ }^{10,11}$. Disproportionately, sJIA contributes about two-thirds of the total mortality rate in JIA ${ }^{12}$. It is an orphan disease with often a chronic course and a major impact on the affected children and their families.

In 1897, while working as a registrar at the Hospital for Sick Children at Great Ormond Street in London, Sir George Frederick Still described a series of patients with three patterns of childhood arthritis, one of which came to be known later as Still's disease, corresponding to what we today call SoJIA $^{13}$. The specific symptoms and frequency of episodes vary from one person to another and the progression of the disorder is difficult to predict. To allow for improved identification and research the International League of Associations of Rheumatology (ILAR) proposed a classification for JIA $^{14}$. To fulfill the criteria for systemic juvenile idiopathic arthritis (sJIA) a child must be under 16 years of age and have "arthritis in one or more joints with or preceded by fever of at least 2 weeks' duration that is documented to be daily ("quotidian") for at least 3 days and accompanied by one or more of the following: (1) evanescent (nonfixed) erythematous rash, (2) generalized lymph node enlargement,

(3) hepatomegaly and/or splenomegaly, serositis". Exclusions include "(a) psoriasis or a history of psoriasis in the patient or a first-degree relative, (b) arthritis in an HLA-B27 positive male beginning after the $6^{\text {th }}$ birthday, (c) ankylosing spondylitis, enthesitis-related arthritis, sacroiliitis with inflammatory bowel disease, Reiter's syndrome, or acute anterior uveitis, or a history of one of these disorders in a first-degree relative, (d) the presence of IgM rheumatoid factor on at least 2 occasions at least 3 months apart"14. According to internationally recognized classification criteria for diagnosis of sJIA the presence of arthritis is mandatory, the diagnosis can only be suspected at disease onset in patients without arthritis. At onset, the prominence of extra-articular features such as spiking fevers, a salmon-colored skin rash, lymphadenopathy and serositis ${ }^{15}$, however, made the diagnosis is often challenging.

Fever is the most common symptom at time of initial presentation. According to Behrens et al., $98 \%$ of patients present with fever ${ }^{15}$, and in a cohort study from the United Kingdom, France, and Spain, 100\% of patients presented with fever ${ }^{16}$. Classically, it has been described as a quotidian fever that spikes to greater than $39^{\circ} \mathrm{C}$ once or twice daily, typically occurring in the evening. While the child is febrile, other symptoms such as arthritis, rash or serositis can worsen and cause significant disturbance of daily life. A persistent fever over 24 hours should suggest other diagnoses, or MAS complicating sJIA $^{15,17}$. Arthritis is the second most common presenting symptom and arthralgia can precede the arthritis ${ }^{18}$. According to Behrens et al., $88 \%$ of children presented with arthritis ${ }^{15}$. In those cases where arthritis was not found initially, it typically appeared within a few months; infrequently the arthritis will not present until several years later ${ }^{10}$. Our 2 patients classically fit in this description showing high grade fever with extra-articular manifestation and fever not responding to antibiotics, but they developed arthritis of multiple joints few months later after initial presentation.

According to Behrens et al. $81 \%$ of patients presented with a rash ${ }^{15}$. Modesto et al., reported that a rash was seen in $89 \%$ of patients with good prognosis and $79 \%$ in patients with bad prognosis ${ }^{16}$. The classical rash is rarely pruritic, evanescent (usually coming and going with fever spikes) and consist of discrete, circumscribed, salmon pink macules $2-10 \mathrm{~mm}$ in size, lesions are more common on the trunk and proximal 
extremities. Stress, warm bath may exacerbate rash and koebner phenomenon is present. Its most diagnostic feature is its transient nature with a group of lesion usually lasting $<1$ hour $^{7}$. Our 1st case presented with this classical rash along with fever. Enlargement of the lymphnodes, the liver and/or the spleen occur in $>70 \%$ of affected children ${ }^{19}$. Our both the cases had hepatospenomgaly and lympadenopathy. In less than $10 \%$ of the patients pericarditis will develop, often asymptomatic and only rarely inducing a tamponade ${ }^{10}$. Pleuritis, pleural effusions, peritonitis are rare manifestation of sJIA can present together with or independently ${ }^{12,17}$. The two major complications of sJIA are MAS and secondary amyloidosis. MAS is a severe, potentially life-threatening complication characterised by the excessive activation of welldifferentiated macrophages, resulting in persistent fever with generalized malaise, hepatosplenomegaly, lymphadenopathy, liver failure, intravascular coagulation, and neurological involvement ${ }^{19,20}$. Ocular manifestations can be seen in sJIA, and uveitis is one of the complications. A case report by Ishihara et al. described a patient with sJIA who developed bilateral panuveitis 3 years after her initial presentation ${ }^{21}$.

The diagnosis of JIA is essentially a clinical one. No laboratory test or combination of studies can confirm the diagnosis of JIA. The main diagnostic challenge for sJIA is the absence of a path gnomonic clinical feature or laboratory marker. In consequence, the clinician has to consider a wide differential diagnosis and perform a large number of investigations to exclude such alternative possibilities. Laboratory and radiological work-up will depend on disease presentation: an atypical clinical picture and in particular the lack of clear arthritis will lead to broader investigations. Many different infections will be considered, such as occult bacterial infection, tuberculosis, brucellosis, Lyme disease, cat scratch disease, infectious mononucleosis. Malignancies, for example leukemia, lymphoma and neuroblastoma, may mimic the symptoms of sJIA, and need to be excluded, if they are clinically suspected, with a bone marrow aspirate. Fever and arthritis may also suggest other inflammatory diseases, like systemic lupus erythematosus, systemic vasculitis, Kawasaki disease, Behçet disease and inflammatory bowel disease. Laboratory investigations will first evaluate the level of inflammation.
Levels of erythrocyte sedimentation rate and C-reactive protein are usually very high. A complete blood count is mandatory to exclude lymphoproliferative diseases; it will typically show an inflammatory anaemia, a leukocytosis with neutrophil predominance and a thrombocytosis. Eleveted ANA titers are present in 40$85 \%$ of oligoarticular and polyarticular JIA, but are rare in sJIA. $5-15 \%$ of polyarticular JIA are seropositive for RF and Anti-cyclic citrullinated peptide antibody is a marker of more aggressive disease. But serodiagnosis of sJIA is almost not possible because sJIA is usually seronegative ${ }^{19}$.

Management of JIA is multidisciplinary involving paediatrician, rheumatologist, physiotherapist and ophthalmologist. Historically, the treatment of sJIA included the use of nonsteroidal antiinflammatory drugs (NSAIDs), if nonresponsive then DMARD (methotrexate, cyclosporine, thalidomide, cyclophosphamide, sulphasalazine, Leflunamide), corticosteroids, Biological agents (Etanercept, Rituximab, Infliximab, Abetacept) and autologous stem cell transplantation ${ }^{19,22-23}$. Our first case initially responded to NSAID, but when presented with polyarthritis then not respond to NSAIDs. Subsequently she was treated with prednisolone and methotrexate, by which her symptoms improved. Our second patient responds well to NSAIDs.

\section{Conclusion}

The diagnosis of a child suspected of having systemic JIA is often difficult, especially at the onset or early in the course of the disease, when the child may have a high spiking fever with evidence of systemic inflammation but no arthritis or other specific signs that allow for a definitive diagnosis. Thus Systemic onset Juvenile Idiopathic arthritis is like an iceberg, the diagnosis of which is usually missed due to lack of definite diagnostic parameter but complication like macrophage activation syndrome, dilated cardiomyopathy may result in morbidity and mortality. In any case of fever of unknown origin we should suspect connective tissue disorder as a possibility especially if not responding to antibiotics.

Conflict of Interest: We have no conflict of interest. 


\section{References}

1. Long SS, Edwards KE. Prolonged, recurrent and periodic fever syndromes. In: Principals and Practice of Pediatric Infectious disease. $4^{\text {th }}$ ed. Elsevier: Churchill Livingstone; 2012, 117-118.

2. Petersdorf RG, Beeson PB. Fever of unexplained origin: report on 100 cases. Medicine Baltimore. 1961; 40: 1-30.

3. Linda SN, Kamat D. Fever without a Focus. In: kliegman RM, Stanton BF, Geme III JWS, Schor NF, Behrman RE, editors. Nelson Textbook of Pediatrics. 20 th ed. Elsevier: Canada; 2016, p1280-1287.

4. Kejariwal D, Sarkar N, Chakraborti SK, et al. Pyrexia of unknown origin: a prospective study of 100 cases. J Postgrad Med. 2001; 47: 104.

5. Pizzo PA, Lovejoy FH, Smith DH. Prolonged fever in children: review of 100 cases. Pediatr. 1975; 55(4): 468-73.

6. Cogulu O, Koturoglu G, Kurugol Z, et al. Evaluation of 80 children with prolonged fever. Pediatr Int. 2003; 45(5): 564-9.

7. Weiss JE, Ilowite NT. Juvenile Idiopathic Arthritis. Pediatr Clin N Am. 2005; 52(2): 413-424.

8. Tolan RW Jr. Fever of unknown origin: a diagnostic approach to this vexing problem. Clin Pediatr (Phila). 2010; 49(3): 207-213.

9. Bleeker-Rovers CP, Vos FJ, de Kleijn EM, et al. A prospective multicenter study on fever of unknown origin: the yield of a structured diagnostic protocol. Medicine (Baltimore). 2007; 86(1): 26-38.

10. Schneider R, Laxer RM. Systemic onset juvenile rheumatoid arthritis. Baillieres Clin Rheumatol. 1998; 12: 245-71.

11. Woo P. Systemic juvenile idiopathic arthritis: Diagnosis, management and outcome. Nat Clin Pract Rheumatol. 2006; 2: 28-34.

12. Wallace CA, Levinson JE. Juvenile rheumatoid arthritis: outcome and treatment for the 1990s. Rheumatic Disease Clinics of North America. 1991; 17(4): 891-905.

13. Still GF. On a form of chronic joint disease in children. Med Chir Trans. 1897; 80: 47-60.

14. Petty RE, Southwood TR, Manners $\mathrm{P}$ et al. International League of Associations for
Rheumatology classification of juvenile idiopathic arthritis: second revision, Edmonton. Journal of Rheumatology. 2004; 31(2): 390-392.

15. Behrens EM, Beukelman T, Gallo L, et al. Evaluation of the presentation of systemic onset juvenile rheumatoid arthritis: Data from the Pennsylvania systemic onset juvenile arthritis registry (Pasojar). J Rheumatol. 2008; 35: 343-8.

16. Modesto C, Woo P, Garc'1a-Consuegra J, et al. Systemic onset juvenile chronic arthritis, polyarticular pattern and hip involvement as markers for a bad prognosis. Clinical and Experimental Rheumatology. 2001; (19)2: 211-217

17. Calabro JJ, Marchesano JM. Fever associated with juvenile rheumatoid arthritis. The New England Journal of Medicine. 1967; 276(1): 11-18.

18. Calabro JJ, Holgerson WB, Sonpal GM, et al. Juvenile rheumatoid arthritis: a general review and report of 100 patients observed for 15 years. Seminars in Arthritis and Rheumatism. 1976; 5(3): 257-298.

19. Wu EY, Bryan AR, Rabinovich CE. Juvenile Idioopathic Arthritis. In: kliegman RM, Stanton BF, Geme III JWS, Schor NF, Behrman RE, editors. Nelson Textbook of Pediatrics. $20^{\text {th }}$ ed. Canada: Elsevier; 2016, 1280-1287.

20. Davi S, Consolaro A, Guseinova D, et al. An international consensus survey of diagnostic criteria for macrophage activation syndrome in systemic juvenile idiopathic arthritis. J Rheumatol. 2011; 38: 764-8.

21. Ishihara $\mathrm{M}$, Nakamura $\mathrm{S}$, Okada $\mathrm{K}$, et al. A case of juvenile rheumatoid arthritis with panuveitis. Journal of Japanese Ophthalmological Society. 1992; 96(1): 109-113.

22. Wallace CA, Sherry DD. Trial of intravenous pulse cyclophosphamide and methylprednisolone in the treatment of severe systemic-onset juvenile rheumatoid arthritis. Arthritis and Rheumatism. 1997; 40(10): 1852-1855.

23. Wulffraat NM, Rooijen EM, Tewarie R, et al. Current perspectives of autologous stem cell transplantation for severe juvenile idiopathic arthritis. Autoimmunity. 2008; 41(8): 632-638. 